\title{
Evaluation of Parameters for Cancer-Induced Sarcopenia in Patients Autopsied after Death from Colorectal Cancer
}

\author{
Hitoshi Ohmoria Isao Kawaharaa,b Takuya Moria,b Shota Nukaga ${ }^{a, b}$ \\ Yi Luo $^{a, c}$ Shingo Kishi ${ }^{a}$ Rina Fujiwara-Tani ${ }^{a}$ Shiori Mori ${ }^{a}$ Kei Goto ${ }^{a}$

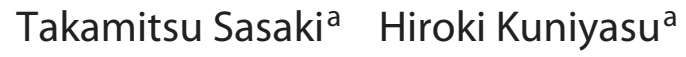 \\ ${ }^{a}$ Department of Molecular Pathology, Nara Medical University, Kashihara, Japan; ${ }^{b}$ Division of Rehabilitation, \\ Hanna Central Hospital, Ikoma, Japan; `Jiangsu Province Key Laboratory of Neuroregeneration, Nantong University, \\ Nantong, China
}

\author{
Keywords \\ Colorectal cancer $\cdot$ Cachexia $\cdot$ Sarcopenia $\cdot$ TNF-a $\cdot$ Myosin \\ light chain
}

\begin{abstract}
Cachexia frequently occurs in cancer patients and is correlated with reduced therapeutic responsiveness and poor prognosis. Although skeletal muscle atrophy is an important factor related to cachexia, biomarkers for its early diagnosis are not yet definitive. In this study, weight loss, body mass index, skeletal muscle index (SMI), serum carcinoembryonic antigen, serum tumor necrosis factor (TNF)- $a$, serum interleukin (IL)-6, serum high mobility group box (HMGB)-1, and SDS-soluble myosin light chain 1 (SDS-MYL1) of the psoas muscle were examined in 8 autopsied cases of death from colorectal cancer (CRC) as biomarkers of cachexia. SDS-MYL1 was positively correlated to SMI and TNF-a was negatively correlated, but the other factors did not show any correlations with SMI. Multivariate analysis showed that of the 3 cytokines, TNF- $a$ and HMGB1 were correlated with SMI. Furthermore, when the biochemical skeletal muscle maturation marker, SDS-MYL1, was compared with serum cytokines, TNF- $a$ and HMGB1 were negatively correlated but IL- 6 was not. In multivariate analysis, only TNF-a was associated with
\end{abstract}

\section{KARGER}

(c) 2019 S. Karger AG, Basel

E-Mail karger@karger.com

www.karger.com/pat
SDS-MYL1. A positive correlation was found between TNF- $a$ and HMGB1. These findings suggest that since TNF-a was inversely correlated with SMI and SDS-MYL1, TNF- $\alpha$ is a serum marker of skeletal muscle atrophy in CRC. Moreover, SDS-MYL1 might be established as a biomarker linked to clinical sarcopenia in experiments in vitro and in vivo.

(c) 2019 S. Karger AG, Basel

\section{Introduction}

Colorectal cancer (CRC) is the third leading cause of cancer-related deaths in Japan, and its incidence has continuously risen [1]. In spite of the advancements in adjuvant therapies, patients with advanced disease still have a poor prognosis [2,3]. One quarter of advanced cases of CRC are associated with liver metastasis, which is a life-threatening event and accounts for 30\% of CRC deaths [4-6].

Cancer cachexia is a condition that impairs quality of life and physical function [7]. Cancer cachexia is found in $40 \%$ of all-stage cancer patients and in $80 \%$ of patients with advanced cancer $[8,9]$. Loss of skeletal muscle vol-

Hitoshi Ohmori and Isao Kawahara contributed equally to this work. 
Table 1. Eight CRC cases and 5 non-cancer cases

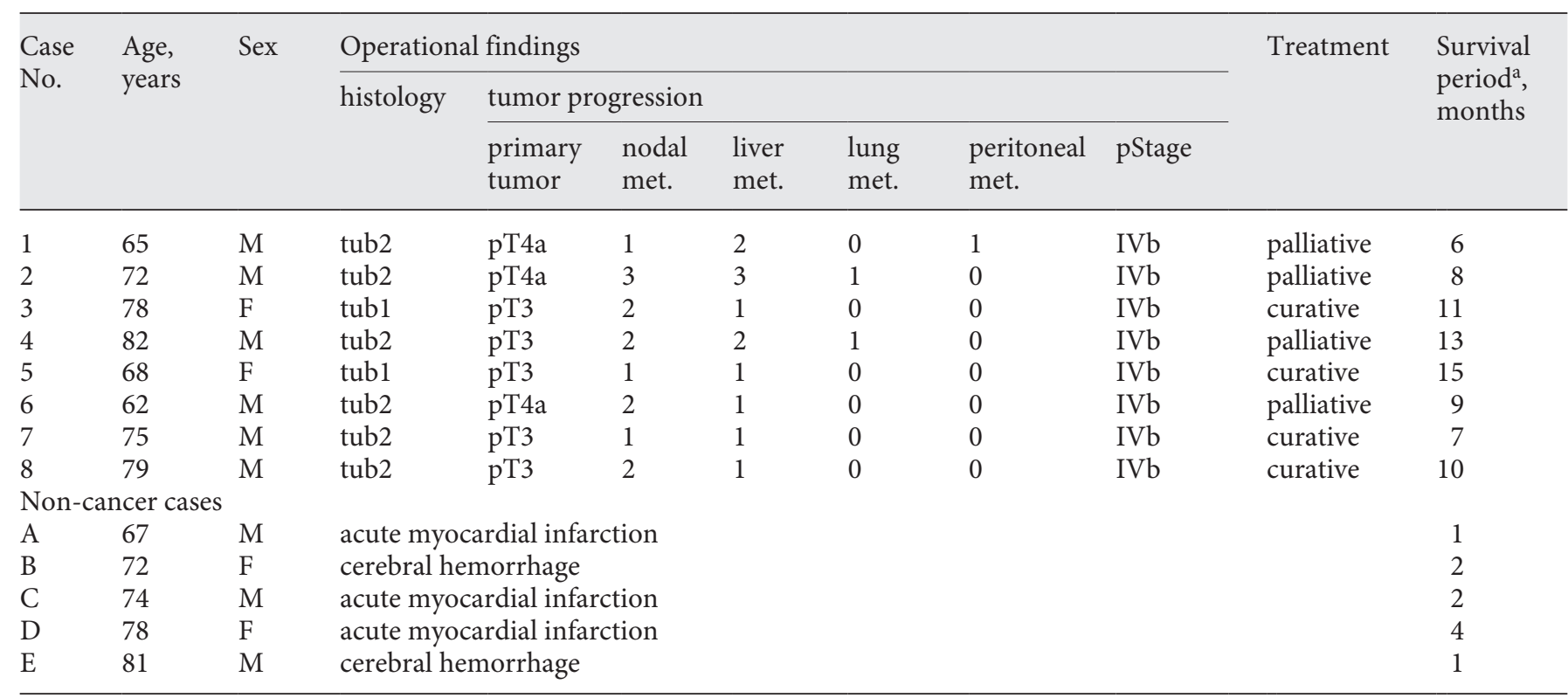

Staging of CRC was done according to the AJCC classification [32].; Primary tumor: pT3, primary tumor invades through the muscularis propria into the pericolorectal tissues; pT4a, primary tumor penetrates to the surface of the visceral peritoneum. Nodal metastasis: pN1, metastasis in 1-3 regional lymph nodes; $\mathrm{pN} 2$, metastasis in $\geq 4$ regional lymph nodes. Liver metastasis: 1 , single foci; 2 , multiple foci. Lung metastasis and peritoneal metastasis: 0 , none; 1, positive. pStage; IVb, any pT and any pN and metastases at $>1$ organ/ site or the peritoneum. M, male; F, female; met., metastasis.

a Postoperative.

ume is an important feature of cachexia [10]. Skeletal muscle atrophy affects tolerability to cancer treatments such as surgery and chemotherapy [11]. Decrease in skeletal muscle mass, i.e., sarcopenia, is associated with shortterm risks of surgery for CRC [12]. For advanced CRC, standard chemotherapies such as folfiri and folfox are effective and are widely indicated $[2,3]$. Skeletal muscle atrophy before or during chemotherapy is correlated with a poor response to treatment $[13,14]$. Sarcopenia is significantly correlated with the poor survival of cancer patients [15-17]. Sarcopenia is also associated with poor survival in end-stage CRC and stage I-III CRC $[18,19]$.

The clinical diagnosis of cancer cachexia used to be based on weight loss, body composition, and physical functioning. Previous studies focused on identifying molecular biomarkers to elucidate the mechanism of cachexia [20]. The need for early diagnosis of cancer cachexia supports the search for biomarkers that reflect the process of skeletal muscle atrophy [21]. However, clinically effective and efficacious biomarkers for making a cancer cachexia diagnosis are still lacking.

In this study, we aimed to identify biomarker candidates for cachexia. We focused on the serum cytokines known to be associated with cachexia and sarcopenia. The inflammatory process evoked by cytokines such as tumor necrosis factor (TNF)- $\alpha$, interleukin (IL)-6 and high mobility group box (HMGB)-1 is considered to play a pivotal role in cachexia and sarcopenia by enhancing anorexia, catabolism, and oxidative stress [11, 22-27]. To judge the significance of these cytokines as biomarkers, their levels were compared with the skeletal muscle index (SMI), a clinical marker of muscle volume [28], and SDSsoluble myosin light chain 1 (SDS-MYL1), a biochemical marker of muscle maturation [29]. SDS-MYL1 is also a candidate marker for sarcopenia and cachexia in in vitro and in vivo models [30]. We investigated whether SDSMYL correlated with clinical sarcopenia parameters as another objective of this study.

\section{Materials and Methods}

\section{Patients}

Of 42 CRC cases (ending in death) diagnosed at the Department of Molecular Pathology, Nara Medical University, from 2001 to 2010, fresh blood and skeletal muscle samples as well as analyzable image and clinical data were available for 8 only. The 8 cases 
Table 2. Clinical aspects of the 8 cancer cases

\begin{tabular}{|c|c|c|c|c|c|c|c|c|c|}
\hline \multirow{2}{*}{$\begin{array}{l}\text { Case } \\
\text { No. }\end{array}$} & \multicolumn{8}{|c|}{ Edmonton symptom assessment scale $[49]^{1}$} & \multirow{2}{*}{$\begin{array}{l}\text { Lower leg } \\
\text { edema }^{2}\end{array}$} \\
\hline & total & drowsiness & nausea & appetite & $\begin{array}{l}\text { shortness } \\
\text { of breath }\end{array}$ & depression & anxiety & well-being & \\
\hline 1 & 56 & 5 & 9 & 10 & 6 & 8 & 9 & 9 & 4 \\
\hline 2 & 41 & 2 & 7 & 7 & 4 & 6 & 7 & 8 & 4 \\
\hline 3 & 34 & 3 & 4 & 8 & 4 & 4 & 4 & 7 & 3 \\
\hline 4 & 46 & 6 & 8 & 8 & 5 & 5 & 6 & 8 & 3 \\
\hline 5 & 36 & 3 & 5 & 6 & 5 & 7 & 4 & 6 & 3 \\
\hline 6 & 37 & 4 & 4 & 6 & 6 & 5 & 5 & 7 & 2 \\
\hline 7 & 38 & 6 & 7 & 6 & 4 & 4 & 4 & 7 & 2 \\
\hline 8 & 38 & 4 & 5 & 7 & 3 & 5 & 7 & 7 & 2 \\
\hline
\end{tabular}

\footnotetext{
${ }^{1}$ Evaluated from the patient complaints described in the medical record.

2 Assessed according to Mosby's Guide to Physical Examination [31]. Pit depth was estimated visually and scored as: $0=$ no clinical edema, $1=$ slight pitting $(2 \mathrm{~mm}$ depth $)$ with no visible distortion, 2 = a somewhat deeper pit $(4 \mathrm{~mm})$ with no readily detectable distortion, 3 = a noticeably deep pit $(6 \mathrm{~mm})$ with the dependent extremity full and swollen, and $4=$ a very deep pit $(8 \mathrm{~mm})$ with the dependent extremity grossly distorted.
}

for which data on cachexia were obtained within 2 months of death were examined (Tables 1, 2). Clinical data (Edmonton Symptom Assessment Scale, body weight, body mass index [BMI], lean body mass $[\mathrm{LBM}]$, serum carcinoembryonic antigen [CEA] concentration, and computed tomography [31]) were obtained from the hospital medical records.

Staging of CRC cases was done according to the American Joint Committee on Cancer Colon and Rectum Cancer Staging [32]. Lymph node metastasis and liver metastasis were observed in all cases, and the stage at the time of surgery was pStage IVb. Curative resection was performed in 4 cases, and palliative surgery was performed in the other 4 . All cases underwent chemotherapy and were administered folfiri or folfox postoperatively, and the survival time after surgery was 6-15 months.

From 112 autopsies of patients whose died from non-cancer causes, 5 were used as controls in this study; only these 5 had adequate samples of fresh blood and skeletal muscle as well as analyzable image and clinical data. Death had occurred within a short time (1-4 months) due to acute myocardial infarction or cerebral hemorrhage. In these cases, no cachexic or sarcopenic changes were observed clinically.

In both the CRC and control cases, autopsy diagnoses did not include severe infections such as sepsis.

\section{Skeletal Muscle Index}

The SMI was calculated as the skeletal muscle area at $\mathrm{L} 3$ in $\mathrm{cm}^{2}$ / (body height in $\mathrm{m})^{2}$. The skeletal muscle area was manually traced using CT images.

\section{Protein Extraction}

Psoas muscle tissue was obtained at autopsy, and then stored at $-80^{\circ} \mathrm{C}$. It was then crushed with a hammer to remove the tendons and fascia. The muscle tissue was washed with cold PBS and pelleted with a sonicator (QSONICA, WakenBtech Co. Ltd., Kyoto, Japan). Whole-cell lysates were prepared as previously described using $0.1 \%$ sodium dodecyl sulfate (SDS)-added radioimmunoprecipitation assay (RIPA) buffer (Thermo Fisher Scientific, Tokyo, Japan) [33]. the protein assay was performed using a Protein Assay Rapid Kit (Wako Pure Chemical Corp., Osaka, Japan).

Blood was obtained from the left cardiac ventricle at autopsy. The blood was incubated undisturbed at room temperature for 20 min and centrifuged at $600 \mathrm{~g}$ for $10 \mathrm{~min}$ at $4^{\circ} \mathrm{C}$. The supernatant (serum) was stored at $-80^{\circ} \mathrm{C}$ till used.

\section{Enzyme-Linked Immunosorbent Assay}

Enzyme-linked immunosorbent assay (ELISA) kits were used to measure the concentration of MYL1 (Cusabio Biotech Co., Ltd., Houston, TX, USA), HMGB1 (Shino-Test Co., Sagamihara, Japan), human TNF- $\alpha$, and IL-6 (Abcam, Cambridge, UK), according to the manufacturers' instructions. For MYL1 measurement, whole-cell lysate was used, whereas for the measurement of HMGB1, TNF- $\alpha$, and IL-6, serum was used.

\section{Statistical Analysis}

Statistical significance was calculated by Spearman $R$ test using InStat software v3.0 (GraphPad Software, Inc., La Jolla, CA, USA). Multiple regression analysis was performed using an EZR program [34]. Data were expressed as the mean \pm standard deviation of 3 independent experiments. $p<0.05$ (two-sided) was considered statistically significant.

\section{Results}

\section{Comparison of Cachexia-Related Markers in CRC and} Non-Cancer Cases

In the $8 \mathrm{CRC}$ and 5 control cases, levels of clinical markers of cachexia (weight loss, BMI, LBM, and SMI), 
Table 3. Comparison of cachexiaassociated parameters between CRC and non-cancer cases

\begin{tabular}{lccc}
\hline & CRC cases, $n=8$ & Non-cancer cases, $n=5$ & $p$ value \\
\hline TNF- $\alpha, \mathrm{pg} / \mathrm{mL}$ & $104 \pm 15$ & $16 \pm 5$ & $<0.0001$ \\
$\mathrm{IL}-6, \mathrm{pg} / \mathrm{mL}$ & $11 \pm 2$ & $2 \pm 1$ & $<0.0001$ \\
$\mathrm{HMGB} 1, \mathrm{pg} / \mathrm{mL}$ & $103 \pm 17$ & $11 \pm 3$ & $<0.0001$ \\
SMI, $\mathrm{cm}^{2} / \mathrm{m}^{2}$ & $36 \pm 5$ & $50 \pm 3$ & 0.0002 \\
Weight loss, kg & $4.3 \pm 2.3$ & $0.36 \pm 0.5$ & 0.0039 \\
BMI, kg/m & $21 \pm 3$ & $28 \pm 3$ & 0.0049 \\
LBM, kg & $44 \pm 5$ & $52 \pm 4$ & 0.0210 \\
SDS-MYL1, pg/g & $18 \pm 4$ & $30 \pm 3$ & 0.0004 \\
CEA, ng/mL & $163 \pm 126$ & $1.2 \pm 0.7$ & 0.017 \\
\hline
\end{tabular}

(1) Serum concentration. (2) Skeletal muscle index (SMI) was calculated as: skeletal muscle area at L3/body height. Skeletal muscle area was measured by computed tomography. (3) Weight loss was calculated as: body weight at operation - body weight at death. (4) Lean body mass $(\mathrm{LBM})$ was calculated as: $0.32810 \times$ body weight $(\mathrm{kg})+0.33929 \times$ height $(\mathrm{cm})-29.5336$ in males, or $0.29569 \times$ body weight $(\mathrm{kg})+0.41813 \times$ height $(\mathrm{cm})$ - 43.2933 in females [50]. (5) SDS-soluble myosin light chain 1 (SDS-MYL1) was measured by ELISA of SDS-soluble whole lysates of the psoas muscles.

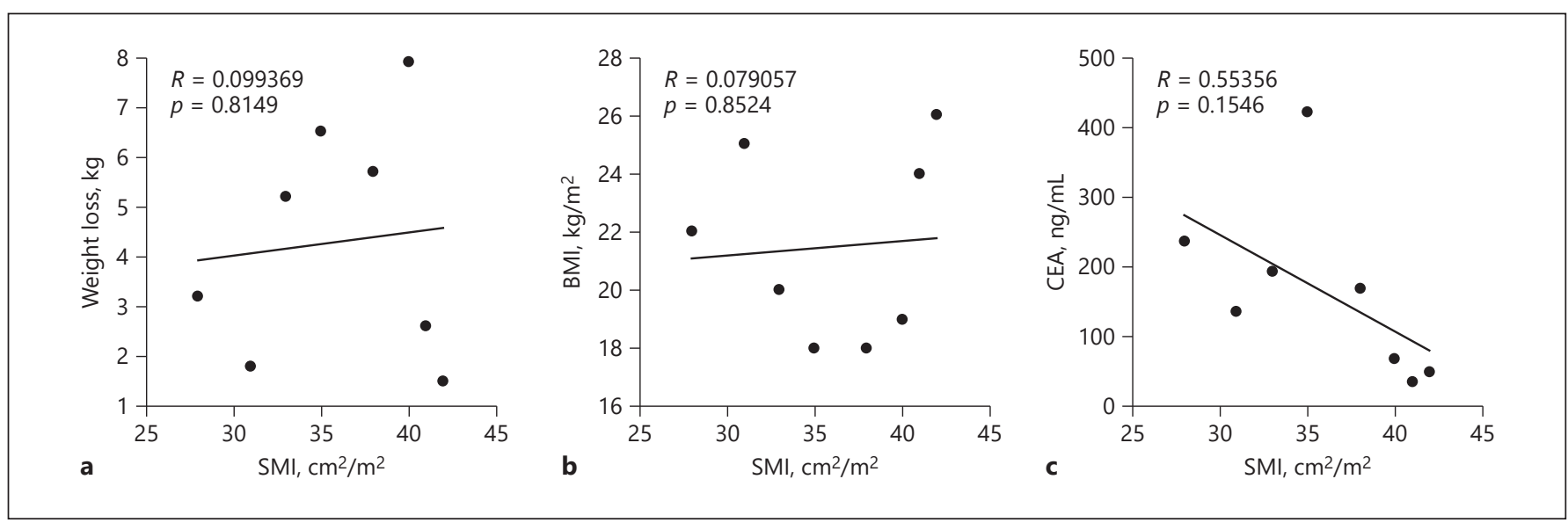

Fig. 1. Relationship between SMI and weight loss, BMI, and serum CEA. Weight loss (a), BMI (b), and serum CEA (c) were compared with SMI. Each parameter was obtained within 2 months of death. The relationship was examined by Spearman regression analysis.

inflammatory cytokines reported to be related to cachexia (TNF, IL-6, and HMGB1), SDS-MYL1 related to skeletal muscle tissue maturity, and CEA related to tumor burden, were compared (Table 3 ).

In CRC cases, weight loss was significantly greater and BMI, LBM, and SMI were significantly reduced compared to the controls. The 3 cytokines were increased 5- to 9-fold in CRC cases compared to controls. In contrast, SDS-MYL1 was reduced in $60 \%$ of the controls. CEA was higher in CRC cases (mean $163 \mathrm{ng} / \mathrm{mL}$ ) than in controls. Thus, both SMI and SDS-MYL1 showed significant differences between CRC and control cases.

\section{Comparison between SMI and Other}

\section{Cachexia-Related Markers in CRC Cases}

Next, we examined the correlation between SMI and other parameters considered to be objective markers of skeletal muscle atrophy. As shown in Figure 1, weight loss, BMI, and CEA showed no significant correlation with SMI. Figure 2 shows that an increase in TNF- $\alpha$ levels was significantly correlated with a decrease in SMI whereas HMGB1 and IL-6 were not correlated with SMI. In contrast, SDS-MYL1 showed a significant positive correlation with SMI. 
Fig. 2. Relationship between SMI, cytokines, and SDS-MYL1. TNF- $\alpha$ (a), IL-6 (b), HMGB1 (c), and SDS-MYL1 (d) levels were compared with SMI. Each parameter was obtained at autopsy. The relationship was examined by Spearman regression analysis.

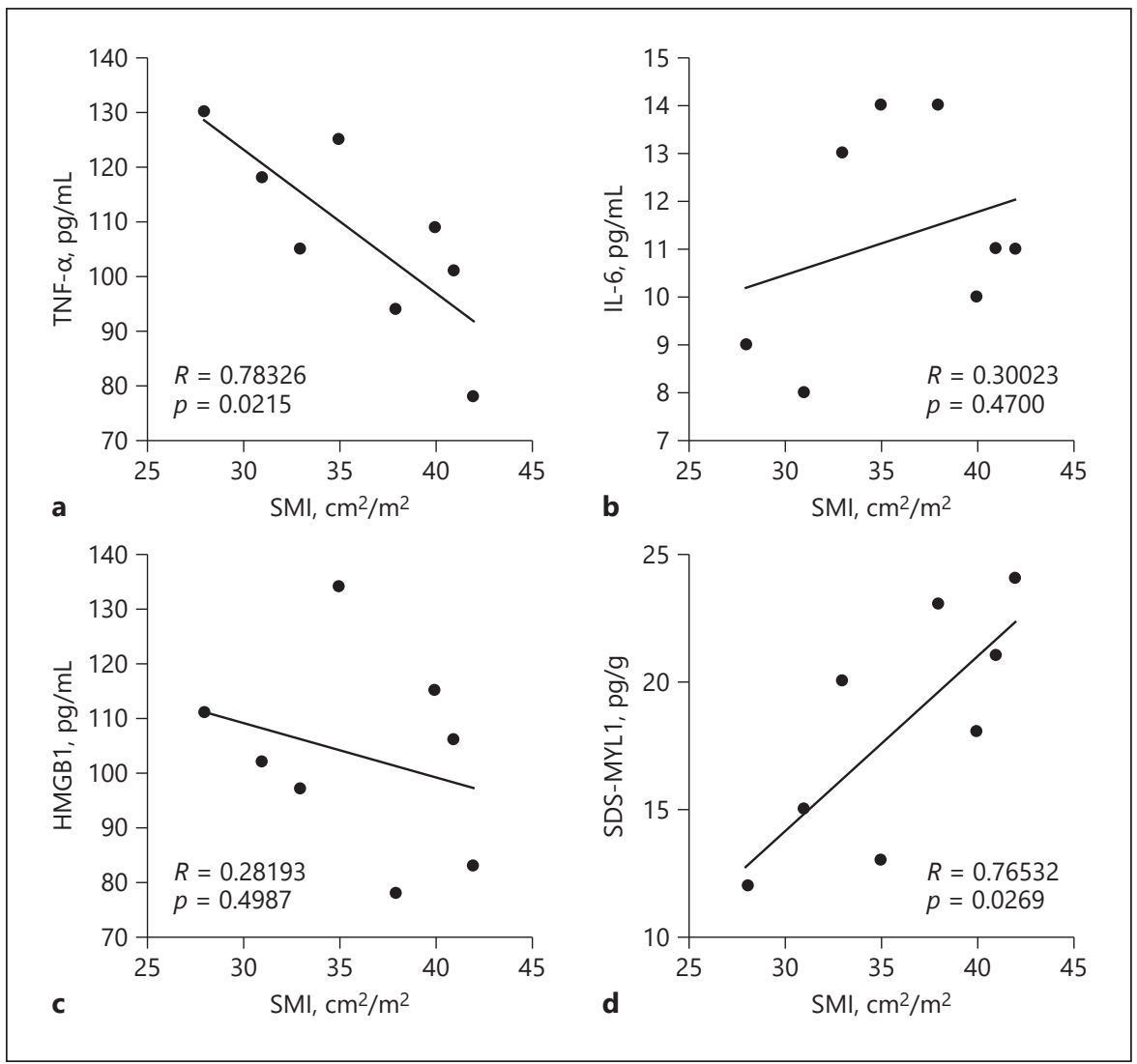

Among these parameters, the correlations between TNF- $\alpha$, IL-6, HMGB1, and SMI were compared using a multiple regression analysis (Table 3 ). TNF- $\alpha$ was most strongly correlated with SMI, followed by HMGB1, whereas no correlation was found between SMI and IL-6.

\section{Relationship of the Three Cytokines in CRC}

Furthermore, when the relationship of the 3 cytokines was examined (Fig. 3), TNF- $\alpha$ and HMGB1 showed a significant positive correlation. Between TNF- $\alpha$ and IL- 6 and between HMGB1 and IL-6, nonsignificant differences were observed.

\section{Comparison between SDS-MYL1 and Cytokines in}

\section{CRC Cases}

Finally, we examined the relationship between SDSMYL1, a biochemical marker of skeletal muscle atrophy, and the 3 cytokines (Fig. 4). Decreased SDS-MYL1 correlated significantly with increased TNF- $\alpha$ and HMGB1, but not with IL-6. The correlations between TNF- $\alpha$, IL-6, HMGB1, and SDS-MYL1 were compared by multiple re- gression analysis (Table 4). Only TNF- $\alpha$ was found to correlate with SDS-MYL1. No correlation was found between SDS-MYL1 and IL-6 or HMGB1.

\section{Discussion}

Skeletal muscle atrophy is considered an important factor in cachexia [10] and to strongly influence the formation of cachexia $[15,16,35,36]$. However, our results showed that SMI, which is a clinical marker of skeletal muscle atrophy, was not significantly correlated with weight loss, BMI, or LBM. This could be because weight loss and BMI involve various factors such as loss of adipose tissue, fluctuation of body fluid such as edema and ascites, and reduction of skeletal muscle volume. Indeed, all cancer cases showed marked edema in the lower legs in this study.

Inflammatory cytokines are deemed to be the cause of skeletal muscle atrophy [22-24]. We compared 3 cytokines, TNF- $\alpha$, HMGB1, and IL- 6 , all reported to be markers of skeletal muscle atrophy $[23,37]$, with SMI and SDSMYL1 which are reported markers of cachexia, and then 


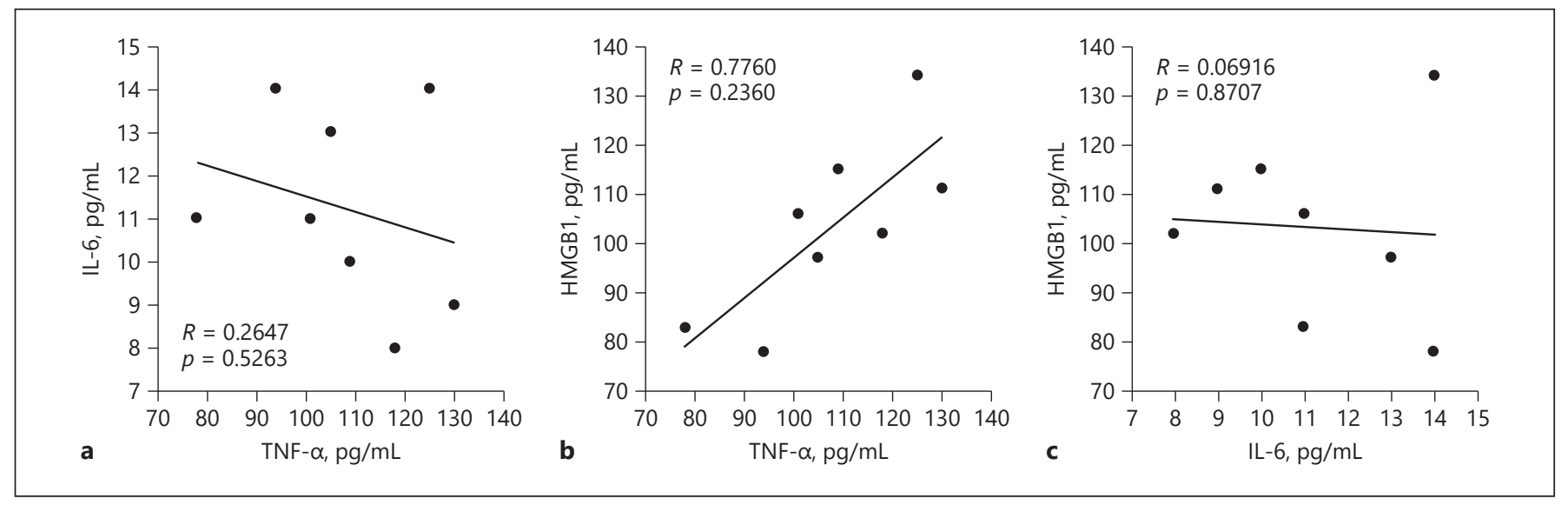

Fig. 3. Relationship among cytokines. TNF- $\alpha$ and IL-6 (a), TNF- $\alpha$ and HMGB1 (b), and IL-6 and HMGB1 (c) were compared. The relationship was examined by Spearman regression analysis.
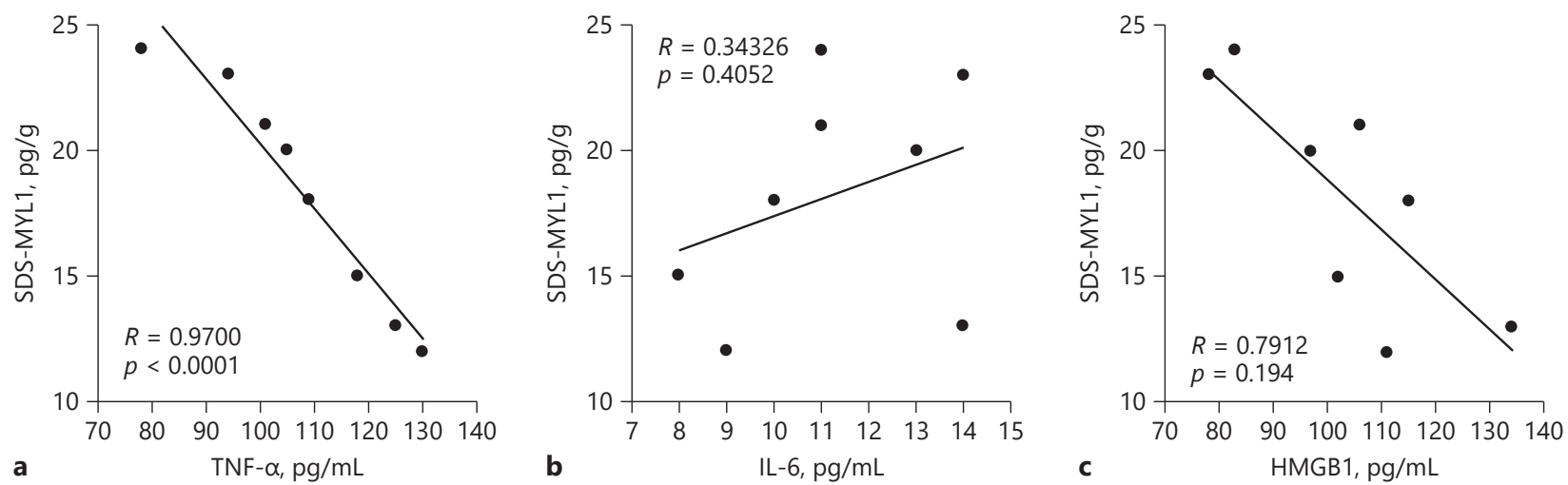

Fig. 4. Relationship between cytokines and SDS-MYL1. TNF- $\alpha$ (a), IL-6 (b), and HMGB1 levels (c) were compared with SDS-MYL1. Each parameter was obtained at autopsy. The relationship was examined by Spearman regression analysis.

Table 4. Multivariate analysis of cytokines

\begin{tabular}{lclll}
\hline & Coefficient & $95 \%$ CI & Standard error & $p$ value \\
\hline SMI & & & & \\
TNF- $\alpha$ & -0.42634181 & -0.66668295 to -0.1860007 & 0.08656434 & 0.007900247 \\
IL-6 & -0.0479042 & -1.19578346 to 1.0999750 & 0.41343488 & 0.913340595 \\
HMGB1 & 0.23434198 & 0.01337157 to 0.4553124 & 0.07958753 & 0.04220148 \\
\hline SDS-MYL1 & & & & \\
TNF- $\alpha$ & -0.22225742 & -0.3530097 to -0.09150517 & 0.0470934 & 0.00917496 \\
IL-6 & 0.22681687 & -0.3976613 to 0.85129503 & 0.22492005 & 0.370295586 \\
HMGB1 & -0.03424226 & -0.1544563 to 0.08597177 & 0.04329783 & 0.473278847 \\
\hline
\end{tabular}

Biomarker for Sarcopenia in Colorectal Cancer
Pathobiology 2019;86:306-314 DOI: $10.1159 / 000503037$ 
examined the biochemical correlations. TNF- $\alpha$ and HMGB1 were found to be correlated with SMI and SDSMYL1, but IL- 6 was not. In contrast, a correlation was found between TNF- $\alpha$ and HMGB. Both TNF- $\alpha$ and HMGB are involved in the activation of innate immunity and proinflammatory feedback [38]. In macrophages, both of these cytokines are known to mutually promote expression and secretion, leading to the exacerbation of inflammation in rheumatoid arthritis and severe infections $[38,39]$.

HMGB is also secreted from colon cancer cells and is known to promote the development of cancer [40-42]. Furthermore, it causes autocrine and paracrine hyperactivation of HMGB1 and TNF- $\alpha$ in inflammatory cells, particularly macrophages [43]. In end-stage cancer patients, abnormal activation of the monocytic system such as hemophagocytosis syndrome may be observed, even when the infection is not clear, suggesting excessive activation of HMGB1 or TNF- $\alpha$ [44, 45]. HMGB1 induces autophagy in skeletal muscle through receptors for advanced glycated end-products and Toll-like receptor 4. Its role in degradation and weight loss in mouse models has been reported [23]. It has also been reported that the inhibition of HMGB1 suppresses skeletal muscle atrophy [46]. In contrast, TNF- $\alpha$ induces autophagy in skeletal muscles through the activation of NF- $\kappa \mathrm{B}$ [47]. The induction of autophagy by inflammatory cytokines is emphasized as a cause of skeletal muscle atrophy. The promotion of autophagy in skeletal muscle atrophy has also been supported by the decrease in SDS-MYL1. Our data did not show any correlation between HMGB1 and SDSMYL1 in univariate or multivariate analysis. HMGB1 has a marked cytokine-activating reaction on the disulfide form [45]. The action of HMGB1 on skeletal muscle is also considered to be more accurate by measuring the disulfide HMGB1.

IL- 6 has been found to be a factor that promotes skeletal muscle atrophy, and TNF- $\alpha$ promotes the expression of IL-6 [47]. However, in our study, no correlation was found between IL- 6 and SMI or SDS-MYL1. This could be because of IL- 6 's bidirectional action on skeletal muscle [48], i.e., IL-6 promotes satellite cell activation and myotube regeneration, but also causes skeletal muscle wasting depending on differences in concentration and duration of action. This could be the reason why no clear association was found between IL- 6 and cachexia or skeletal muscle atrophy in this study.

Cachexia and sarcopenia are extremely clinical concepts, and how to extrapolate the results of animal models and cell experiments to humans is an important issue. In this study, although the number of cases was small, it was possible to clearly show the associations among SDSMYL1, SMI, and cytokines. SDS-MYL1 is associated with skeletal muscle maturation [29] and reflects sarcopenia in mouse cachexia models [30]. The fact that SDS-MYL1 correlated well with SMI in this study is an important finding, suggesting that the results of SDS-MYL1 can be extrapolated to clinical sarcopenia in animal models and culture cell experiments.

In conclusion, our study results showed that serum TNF- $\alpha$ levels can be considered effective biomarkers for skeletal muscle atrophy. Furthermore, the interaction between TNF- $\alpha$ and HMGB1 is thought to induce skeletal muscle atrophy, so targeting this interaction could be useful for the prevention and amelioration of cancerous skeletal muscle atrophy and thus cancerous cachexia. In this study, a small number of cases had to be examined due to the lack of biosamples and clinical data. In the future, it is hoped that the interest in cachexia and sarcopenia in cancer patients will deepen and studies using more cases will be possible.

\section{Acknowledgements}

The authors thank Ms. Tomomi Masutani for expert assistance with the preparation of this manuscript.

\section{Statement of Ethics}

Written informed consent was obtained from the patients' families for their participation in the study, and all identifying information was removed from patient samples prior to their analysis to ensure strict privacy protection (unlinkable anonymization). All procedures were performed in accordance with the Ethics Guidelines for Human Genome/Gene Research enacted by the Japanese Government and with the approval of the Ethics Committee of Nara Medical University (approval No. 937).

\section{Disclosure Statement}

The authors have no conflict of interest to declare.

\section{Funding Sources}

This work was supported by MEXT KAKENHI Grant Number 16H05164 (HK), 17K15648 (RFT), 17K19923 (HK), 18K16671 (SK), 18K17726 (IK), 18K10788 (KF). 


\section{References}

1 Wakao F, Nishimoto H, Kataonoda K, Tsukuma H, Mikami H, editors. Cancer Statistics in Japan, 2013. Tokyo: National Cancer Research Institute; 2013.

2 Sánchez-Gundín J, Fernández-Carballido AM, Martínez-Valdivieso L, Barreda-Hernández D, Torres-Suárez AI. New trends in the therapeutic approach to metastatic colorectal cancer. Int J Med Sci. 2018 Apr; 15(7):659-65.

3 Watanabe T, Muro K, Ajioka Y, Hashiguchi Y, Ito Y, Saito Y, et al.; Japanese Society for Cancer of the Colon and Rectum. Japanese Society for Cancer of the Colon and Rectum (JSCCR) guidelines 2016 for the treatment of colorectal cancer. Int J Clin Oncol. 2018 Feb; 23(1):1-34

4 Kuniyasu H, Luo Y, Fujii K, Sasahira T, Moriwaka Y, Tatsumoto N, et al. CD10 enhances metastasis of colorectal cancer by abrogating the anti-tumoural effect of methionine-enkephalin in the liver. Gut. 2010 Mar;59(3): 348-56.

5 Fujimoto Y, Nakanishi Y, Sekine S, Yoshimura K, Akasu T, Moriya Y, et al. CD10 expression in colorectal carcinoma correlates with liver metastasis. Dis Colon Rectum. 2005 Oct; 48(10):1883-9.

6 Fong Y, Kemeny N, Paty P, Blumgart LH, Cohen AM. Treatment of colorectal cancer: hepatic metastasis. Semin Surg Oncol. 1996 JulAug;12(4):219-52.

7 Donohoe CL, Ryan AM, Reynolds JV. Cancer cachexia: mechanisms and clinical implications. Gastroenterol Res Pract. 2011;2011: 601434.

8 Bozzetti F; SCRINIO Working Group. Screening the nutritional status in oncology: a preliminary report on 1,000 outpatients. Support Care Cancer. 2009 Mar; 17(3):27984.

9 Paillaud E, Caillet P, Campillo B, Bories PN. Increased risk of alteration of nutritional status in hospitalized elderly patients with advanced cancer. J Nutr Health Aging. 2006 Mar-Apr;10(2):91-5.

10 Evans WJ, Morley JE, Argilés J, Bales C, Bara$\cos \mathrm{V}$, Guttridge D, et al. Cachexia: a new definition. Clin Nutr. 2008 Dec;27(6):793-9.

11 Fearon K, Arends J, Baracos V. Understanding the mechanisms and treatment options in cancer cachexia. Nat Rev Clin Oncol. 2013 Feb;10(2):90-9.

12 Yang J, Zhang T, Feng D, Dai X, Lv T, Wang $\mathrm{X}$, et al. A new diagnostic index for sarcopenia and its association with short-term postoperative complications in patients undergoing surgery for colorectal cancer. Colorectal Dis. 2019 May;21(5):538-47.
13 Sasaki S, Oki E, Saeki H, Shimose T, Sakamoto $\mathrm{S}, \mathrm{Hu} \mathrm{Q}$, et al. Skeletal muscle loss during systemic chemotherapy for colorectal cancer indicates treatment response: a pooled analysis of a multicenter clinical trial (KSCC 1605A). Int J Clin Oncol. 2019 Oct;24(10):120413.

14 Miyamoto Y, Hiyoshi Y, Akiyama T, Kiyozumi Y, Eto K, Yohei N, et al. Low skeletal muscle mass before salvage-line chemotherapy is a poor prognostic factor in patients with refractory metastatic colorectal cancer. Digestion. 2019;99(1):79-85.

15 Shachar SS, Williams GR, Muss HB, Nishijima TF. Prognostic value of sarcopenia in adults with solid tumours: A meta-analysis and systematic review. Eur J Cancer. 2016 Apr;57:58-67.

16 Zhou X, Wang JL, Lu J, Song Y, Kwak KS, Jiao $\mathrm{Q}$, et al. Reversal of cancer cachexia and muscle wasting by ActRIIB antagonism leads to prolonged survival. Cell. 2010 Aug;142(4): 531-43

17 Deng CY, Lin YC, Wu JS, Cheung YC, Fan $\mathrm{CW}$, Yeh KY, et al. Progressive sarcopenia in patients with colorectal cancer predicts survival. AJR Am J Roentgenol. 2018 Mar;210(3): 526-32.

18 Brown JC, Caan BJ, Meyerhardt JA, Weltzien E, Xiao J, Cespedes Feliciano EM, et al. The deterioration of muscle mass and radiodensity is prognostic of poor survival in stage I-III colorectal cancer: a population-based cohort study (C-SCANS). J Cachexia Sarcopenia Muscle. 2018 Aug;9(4):664-72.

19 Choi MH, Oh SN, Lee IK, Oh ST, Won DD. Sarcopenia is negatively associated with longterm outcomes in locally advanced rectal cancer. J Cachexia Sarcopenia Muscle. 2018 Feb; 9(1):53-9.

20 Bruggeman AR, Kamal AH, LeBlanc TW, Ma JD, Baracos VE, Roeland EJ. Cancer cachexia: beyond weight loss. J Oncol Pract. 2016 Nov; 12(11):1163-71.

21 Loumaye A, Thissen JP. Biomarkers of cancer cachexia. Clin Biochem. 2017 Dec;50(18): 1281-8.

22 Patel HJ, Patel BM. TNF- $\alpha$ and cancer cachexia: molecular insights and clinical implications. Life Sci. 2017 Feb;170:56-63.

23 Luo Y, Yoneda J, Ohmori H, Sasaki T, Shimbo $\mathrm{K}$, Eto S, et al. Cancer usurps skeletal muscle as an energy repository. Cancer Res. 2014 Jan; 74(1):330-40.

24 Lucia S, Esposito M, Rossi Fanelli F, Muscaritoli M. Cancer cachexia: from molecular mechanisms to patient's care. Crit Rev Oncog. 2012;17(3):315-21.

25 Bjorklund G, Dadar M, Aaseth J, Chirumbolo $\mathrm{S}$, Pen JJ. Cancer-associated cachexia, reactive oxygen species, and nutrition therapy. Curr Med Chem. 2018. DOI: 10.2174/0929867325 666180629123817.
26 Shyh-Chang N. Metabolic changes during cancer cachexia pathogenesis. Adv Exp Med Biol. 2017; 1026:233-49.

27 Ezeoke CC, Morley JE. Pathophysiology of anorexia in the cancer cachexia syndrome. J Cachexia Sarcopenia Muscle. 2015 Dec;6(4): 287-302.

28 Choi Y, Oh DY, Kim TY, Lee KH, Han SW, Im SA, et al. Skeletal muscle depletion predicts the prognosis of patients with advanced pancreatic cancer undergoing palliative chemotherapy, independent of body mass index. PLoS One. 2015 Oct;10(10):e0139749.

29 Yazaki Y, Mochinaga S, Raben MS. Fractionation of the light chains from rat and rabbit cardiac myosin. Biochim Biophys Acta. 1973 Dec;328(2):464-9.

30 Mori T, Ohmori H, Luo Y, Mori S, Miyagawa Y, Nukaga S, et al. Giving combined mediumchain fatty acids and glucose protects against cancer-associated skeletal muscle atrophy. Cancer Sci. 2019;110(10):3391-9.

31 Seidel HM, Ball JW, Dains JE, Benedict GW. Heart and blood vessels. In: Schrefer S, editor. Mosby's guide to physical examination. St. Louis (MO): Mosby; 1995. p. 419.

32 Edge S, Byrd DR, Compton CC, Fritz AG, Greene FL, Trotti A. AJCC cancer staging manual. 7th ed. New York: Springer; 2010.

33 Kuniyasu H, Oue N, Wakikawa A, Shigeishi H, Matsutani N, Kuraoka K, et al. Expression of receptors for advanced glycation endproducts (RAGE) is closely associated with the invasive and metastatic activity of gastric cancer. J Pathol. 2002 Feb;196(2):163-70.

34 Kanda Y. Investigation of the freely available easy-to-use software 'EZR' for medical statistics. Bone Marrow Transplant. 2013 Mar; 48(3):452-8.

35 Fearon KC. Cancer cachexia: developing multimodal therapy for a multidimensional problem. Eur J Cancer. 2008 May;44(8):1124-32.

36 Acharyya S, Butchbach ME, Sahenk Z, Wang H, Saji M, Carathers M, et al. Dystrophin glycoprotein complex dysfunction: a regulatory link between muscular dystrophy and cancer cachexia. Cancer Cell. 2005 Nov;8(5):421-32.

37 Prado BL, Qian Y. Anti-cytokines in the treatment of cancer cachexia. Ann Palliat Med. 2019 Jan;8(1):67-79.

38 Bao GQ, He L, Lee D, D’Angelo J, Wang HC. An ongoing search for potential targets and therapies for lethal sepsis. Mil Med Res. 2015 Aug;2(1):20.

39 Andersson U, Erlandsson-Harris H. HMGB1 is a potent trigger of arthritis. J Intern Med. 2004 Mar;255(3):344-50.

40 Ohmori H, Luo Y, Kuniyasu H. Non-histone nuclear factor HMGB1 as a therapeutic target in colorectal cancer. Expert Opin Ther Targets. $2011 \mathrm{Feb}$;15(2):183-93. 
41 Kuniyasu H, Chihara Y, Kondo H. Differential effects between amphoterin and advanced glycation end products on colon cancer cells. Int J Cancer. 2003 May; 104(6):722-7.

42 Kuniyasu H, Chihara Y, Takahashi T. Co-expression of receptor for advanced glycation end products and the ligand amphoterin associates closely with metastasis of colorectal cancer. Oncol Rep. 2003 Mar-Apr;10(2):4458.

43 Rojas A, Delgado-López F, Perez-Castro R, Gonzalez I, Romero J, Rojas I, et al. HMGB1 enhances the protumoral activities of M2 macrophages by a RAGE-dependent mechanism. Tumour Biol. 2016 Mar;37(3):3321-9.
44 Billiau AD, Roskams T, Van Damme-Lombaerts R, Matthys P, Wouters C. Macrophage activation syndrome: characteristic findings on liver biopsy illustrating the key role of activated, IFN- $\gamma$-producing lymphocytes and IL-6- and TNF- $\alpha$-producing macrophages. Blood. 2005 Feb;105(4):1648-51.

45 Palmblad K, Schierbeck H, Sundberg E, Horne AC, Harris HE, Henter JI, et al. High systemic levels of the cytokine-inducing HMGB1 isoform secreted in severe macrophage activation syndrome. Mol Med. 2015 Jan;20(1):538-47.

46 Kawahara I, Goto K, Kodama K, Luo Y, Fujiwara-Tani R, Mori T, et al. Magnetic hyperthermia using self-controlled heating elements consisting of $\mathrm{Fe}-\mathrm{Al}$ milling alloy induces cancer cell apoptosis while preserving skeletal muscle. Pathobiology. Forthcoming 2019.
47 Yeo D, Kang C, Zhang T, Ji LL. Avenanthramides attenuate inflammation and atrophy in muscle cells. J Sport Health Sci. 2019 Mar; 8(2):189-95.

48 Belizário JE, Fontes-Oliveira CC, Borges JP, Kashiabara JA, Vannier E. Skeletal muscle wasting and renewal: a pivotal role of myokine IL-6. Springerplus. 2016 May;5(1):619.

49 Bruera E, Kuehn N, Miller MJ, Selmser P, Macmillan K. The Edmonton Symptom Assessment System (ESAS): a simple method for the assessment of palliative care patients. J Palliat Care. 1991;7(2):6-9.

50 Hume R. Prediction of lean body mass from height and weight. J Clin Pathol. 1966 Jul; 19(4):389-91. 